\title{
Near-atomic resolution CryoEM analysis of the Type III Secretion Injectisome
}

Natalie C. J. Strynadka

Department of Biochemistry, 2350 Health Sciences Mall, University Of British Columbia, Vancouver, Canada V6T $1 Z 3$

E-mail:ncjs@mail.ubc.ca

Bacteria have evolved several sophisticated assemblies to transport proteins across their biological membrane, including those required specifically for pathogenicity. Recent advances in our understanding of the molecular details governing the molecular action of these protein secretion systems has benefited from an integrated x-ray crystallography, NMR, mass spectroscopy, electron microscopy, and molecular modeling toolbox. Highlights of recent advances in our piece wise structure/function analysis of the multi-membrane spanning Type III Secretion system "injectisome" will be presented with particular emphasis on new insights provided by single particle cryoEM methods. A molecular understanding of the Type III systems being garnered from these studies furthermore provides the foundation for the development of new classes of antibacterials and vaccines to combat infection in the clinic and community. 